\title{
Dead or alive: DEAD-box ATPases as regulators of ribonucleoprotein complex condensation
}

\section{Review Article}

Author(s):

Weis, Karsten

Publication date:

2021-04

Permanent link:

https://doi.org/10.3929/ethz-b-000482471

Rights / license:

In Copyright - Non-Commercial Use Permitted

Originally published in:

Biological Chemistry 402(5), https://doi.org/10.1515/hsz-2020-0381

\section{Funding acknowledgement:}

179275 - Structure and Function of the Nuclear Pore Complex (SNF)

193740 - CRSII5_193740 / 1 (SNF) 


\section{Review}

\section{Karsten Weis* \\ Dead or alive: DEAD-box ATPases as regulators of ribonucleoprotein complex condensation}

https://doi.org/10.1515/hsz-2020-0381

Received December 2, 2020; accepted March 2, 2021; published online April 2, 2021

\begin{abstract}
DEAD-box ATPase proteins are found in all clades of life and have been associated with a diverse array of RNA-processing reactions in eukaryotes, bacteria and archaea. Their highly conserved core enables them to bind RNA, often in an ATP-dependent manner. In the course of the ATP hydrolysis cycle, they undergo conformational rearrangements, which enable them to unwind short RNA duplexes or remodel RNA-protein complexes. Thus, they can function as RNA helicases or chaperones. However, when their conformation is locked, they can also clamp RNA and create ATP-dependent platforms for the formation of higher-order ribonucleoprotein complexes. Recently, it was shown that DEAD-box ATPases globally regulate the phaseseparation behavior of RNA-protein complexes in vitro and control the dynamics of RNA-containing membraneless organelles in both pro- and eukaryotic cells. A role of these enzymes as regulators of RNA-protein condensates, or 'condensases', suggests a unifying view of how the biochemical activities of DEAD-box ATPases are used to keep cellular condensates dynamic and 'alive', and how they regulate the composition and fate of ribonucleoprotein complexes in different RNA processing steps.
\end{abstract}

Keywords: biomolecular condensates; DEAD-box ATPases; liquid-liquid phase separation; membraneless organelles; ribonucleoprotein complexes; RNA processing.

\section{Introduction: structural overview of the DEAD-box ATPase cycle}

DEAD-box ATPases are ubiquitously expressed in both proand eukaryotic cells, and are even present in the genome of

*Corresponding author: Karsten Weis, Institute of Biochemistry, ETH Zurich, Otto-Stern-Weg 3, CH-8093 Zurich, Switzerland,

E-mail: karsten.weis@bc.biol.ethz.ch. https://orcid.org/0000-00017224-925X many viruses. About 26 DEAD-box proteins can be found in budding yeast whereas 38 proteins of this protein family are present in the human proteome (Jarmoskaite and Russell 2014; Linder and Jankowsky 2011; Ozgur et al. 2015). In many cases, DEAD-box proteins are highly abundant, and are expressed at micromolar concentrations in cells, comparable to ribosomal proteins or copious metabolic enzymes. They function in all aspects of RNA processing ranging from transcription, editing, mRNA splicing, nuclear export, translation, localization, quality control, degradation to ribosome biogenesis (Hooper and Hilliker 2013; Jarmoskaite and Russell 2014; Linder and Jankowsky 2011; Ozgur et al. 2015).

DEAD-box ATPases are characterized by a highly conserved core consisting of two structurally very similar RecA domains, named after their homology to the bacterial recombination factor RecA (Caruthers and McKay 2002). The two RecA domains contain all the critical sequence elements that mediate ATP and RNA binding (Figure 1) (reviewed in Linder and Jankowsky [2011]; Ozgur et al. [2015]; and Jarmoskaite and Russell [2014]). In the active conformation, ATP binds within a cleft that is formed between the $\mathrm{N}$ - and C-terminal RecA domain positioning the eponymous aspartate-glutamate-alanineaspartate (DEAD) motif in proximity to the gammaphosphate to form a catalytically competent ATPase site. On the opposing side of the structure, a surface lined with positively charged amino acid residues constitutes the RNA binding pocket that stretches across both RecA domains and accommodates five or more nucleotides adopting a slightly kinked conformation. Since there are no direct contacts with the RNA bases, only with the backbone, many DEAD-box proteins show little sequence specificity in their interactions with RNA (Jarmoskaite and Russell 2014; Linder and Jankowsky 2011; Ozgur et al. 2015). Yet, selectivity for specific RNAs, or classes of RNA, can be mediated by additional domains or co-factors, or by a specific subcellular localization, which may restrict the access to substrates (see also below). The RNA binding region of DEAD-box proteins can also accommodate double-stranded RNA, which could be critical for their helicase activity. 
The $\mathrm{N}$ - and C-terminal RecA domains are connected by a flexible linker. In the absence of RNA and ATP, the relative orientation of the two RecA domains to each other is often very flexible, and DEAD-box proteins adopt an 'open' conformation. Whereas the relative orientation of the two RecA domains can vary in the inactive, open state, all DEAD-box proteins display a very similar conformational arrangement upon binding of ATP and RNA. In the active, 'closed' state, the two RecA domains are positioned to form a hydrolysis-competent ATP binding pocket and at the same time the positively-charged RNA binding surfaces on the RecA domains are perfectly aligned (reviewed in Hilbert et al. [2009] and Ozgur et al. [2015]). Given the malleable orientation of the two RecA domains to each other, binding of ATP and RNA generally favors the formation of this closed state, which leads to a coordination of ATP and RNA binding. This also explains why DEAD-box proteins can function as ATP-dependent RNA binding proteins and why RNA stimulates ATP hydrolysis (reviewed in Jarmoskaite and Russell [2014]; Linder and Jankowsky [2011]; and Ozgur et al. [2015]).

Upon ATP hydrolysis, release of ADP, $\mathrm{P}_{\mathrm{i}}$ and RNA restores the flexibility of the two RecA domains promoting the opening of the structure and completing the ATPase cycle. The large structural changes that occur during this cycle -together with the RNA kink that is induced upon binding-can explain the biochemical function of DEAD-box ATPases as unwinders of short RNA duplexes or as RNP remodelers. Yet, in the absence of substrate release, the two RecA cores can also remain locked in the closed conformation, hold onto the RNA and recruit other proteins to the RNA to form a stable ribonucleoprotein complex (reviewed in Jarmoskaite and Russell [2014]; Linder and Jankowsky [2011]; and Ozgur et al. [2015]).

\section{ATPase activating proteins for DEAD-box proteins}

The substantial structural rearrangements that are observed in the course of the ATPase cycle help to rationalize why DEAD-box proteins are often inefficient enzymes on their own. Stable interactions with RNA and ATP only occur when the flexible core structure is in the closed conformation decreasing the on-rate for substrate binding. At the same time, this suggests that release of RNA and $\mathrm{ADP} / \mathrm{P}_{\mathrm{i}}$ is also slow since interaction with ADP and RNA on both RecA domains stabilize a closed state. Indeed, $P_{i}$ release was found to be rate-limiting for several DEAD-box ATPases, and thus the actual ATP hydrolysis step is not necessarily the reason for the slow reaction cycle of these enzymes compared with other ATPases (Cao et al. 2011; Henn et al. 2008; Hilbert et al. 2010; Russell et al. 2014; Wong et al. 2016). To overcome bottlenecks in the reaction cycle, DEAD-box ATPases interact with activating co-

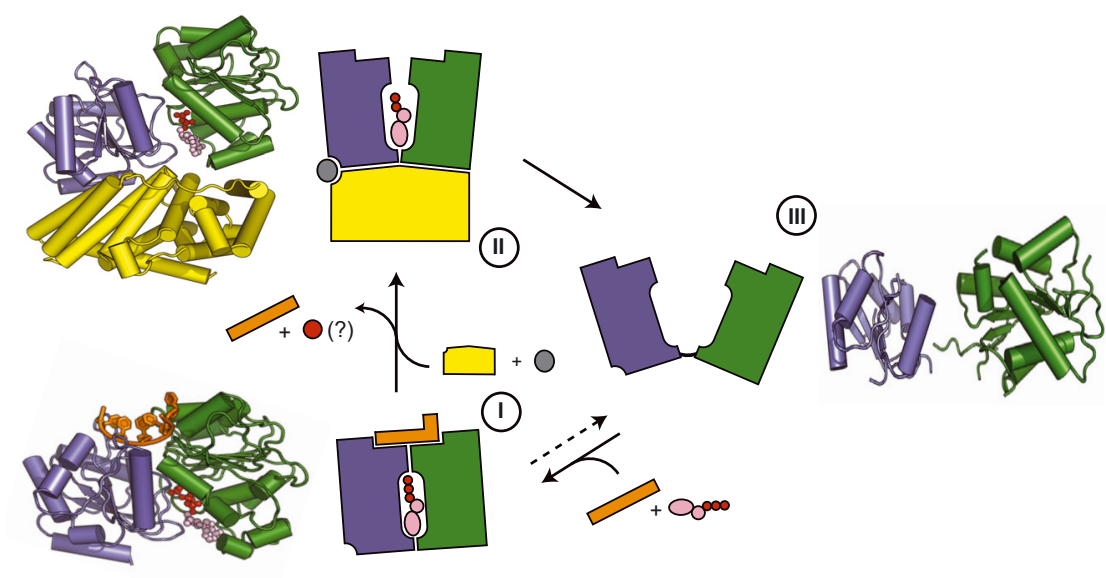

Gle1-MIF4G $\square$ Dbp5-RecA-N 5 Dbp5-RecA-C $\left[7\right.$ Adenosine $\bigcirc$ Phosphate $\bigcirc \quad R N A \square \quad I_{6} \bigcirc$

Figure 1: Model of the DEAD-box ATPase cycle.

The model is based on structural analyses of yeast Dbp5 and its MIF4G-activator Gle1 (Montpetit et al. 2011). In the presence of ATP, the DEAD-box protein binds RNA (state I). The activator binds both the C-terminal (RecA-C) and N-terminal RecA domains (RecA-N), separating the two RecA-like domains to promote substrate release (state II). Subsequent substrate release can cause the two RecA domains to further separate (state III). In the presence of an activator, the DEAD-box protein can likely toggle between the conformational states I and II preventing the off-state III. For details see text. Please note that the interaction between Dbp5 and Gle1 is promoted by IP6, which is a unique feature of this DEAD-box/MIF4G pair. 
factors. An important group of ATPase activating proteins contain MIF4G (middle domain of eukaryotic initiation factor 4G)-like domains (Table 1). Despite their very limited sequence homology, structurally and biochemically characterized MIF4G activators contain $\alpha$-helical HEAT repeats that make very similar contacts with both RecA domains and bind to DEAD-box proteins on the opposing side of the RNA away from the ATP binding cleft (Chen et al. 2014; Mathys et al. 2014; Montpetit et al. 2011; Oberer et al. 2005; Pühringer et al. 2020; Schuller et al. 2020; Schütz et al. 2008). This position does not allow them to directly stimulate ATP hydrolysis. Instead, critical for the activation are a primary, stable interaction between the MIF4G protein and the C-terminal RecA domain, as well as a secondary, transient interaction with the N-terminal RecA domain. When both binding sites are engaged, DEAD-box proteins adopt a partially open state, in which the RNA binding surface is rearranged preventing the formation of the localized pocket of positive charges significantly lowering the affinity for RNA. However, the DEAD-box protein can readily go back to the fully active, closed conformation and once again bind to RNA and ATP, simply by detaching the transient, low-affinity interaction that forms between the MIF4G protein and the N-terminal RecA domain. Thus, in complex with MIF4G activators, DEAD-box proteins can rapidly toggle between two states, a catalytically active state and a state that promotes substrate release. Hence, MIF4G activators speed up the reaction cycle by restricting the conformational space that the two RecA domains can occupy and by preventing the formation of fully inactive orientations (reviewed in Ozgur et al. 2015).

A strong dependence on specific activators allows for additional layers of regulation, which might be particularly critical for those DEAD-box proteins which by themselves do not show selectivity for their RNA substrates. For example, to avoid promiscuous engagement with cellular RNAs, MIF4G activators and other partners could help mediate interactions with specific RNAs or locally activate DEAD-box proteins to spatially restrict their enzymatic

Table 1: Examples of MIF4G domain-containing regulators of DEAD-box ATPases.

\begin{tabular}{llll}
\hline MIF4G protein & DEAD-box protein & & \\
\hline elF4G & elF4A & Activator & Schütz et al. (2008) \\
Gle1 & Dbp5 & Activator & Montpetit et al. (2011) \\
NOT1 & DDX6/Dhh1 & Activator & Mathys et al. (2014) \\
& & Mugler et al. (2016) \\
UAP56/Sub2 & THOC/Tho2 & Activator & $\begin{array}{l}\text { Pühringer et al. (2020) } \\
\text { Schuller et al. (2020) }\end{array}$ \\
CWC22 & elF4AIII & Inhibitor & Barbosa et al. (2012) \\
\hline
\end{tabular}

activity. One example for this is the MIF4G protein Gle1, which is a potent activator of the DEAD-box protein Dbp5/ DDX19 (Alcazar-Roman et al. 2006; Weirich et al. 2006). Gle1 is bound to the nuclear pore complex, thereby restricting Dbp5/DDX19's activity to this cellular location, where it can function in the directional export of RNAs out of the nucleus (Alcazar-Roman et al. 2006; Folkmann et al. 2011; Weirich et al. 2006).

Interestingly, MIF4G proteins can also inhibit the ATPase cycle of DEAD-box ATPases (Table 1). One example is the MIF4G domain of CWC22, which holds its DEAD-box partner eIF4AIII in an open conformation inhibiting the interaction with ATP and RNA (Barbosa et al. 2012; Buchwald et al. 2013). An alternative mode of inhibition is illustrated by the proteins Mago-Y14, which stabilize the closed conformation of eIF4AIII, thus preventing the release of substrates and arresting the enzyme in the RNA, ADP and Pi-bound state (C. B. F. Andersen et al. 2006; Bono et al. 2006; Nielsen et al. 2009). This allows for the formation of a stable exon junction complex (EJC) that is deposited onto mRNA as a consequence of a nuclear splicing event, and subsequently accompanies mRNAs to the cytoplasm (Le Hir et al. 2000).

\section{DEAD-box proteins contain flexible extensions that can promote phase separation}

In addition to the highly conserved core domain, almost all DEAD-box proteins contain variable extensions at their $\mathrm{N}$ and C-termini (Jarmoskaite and Russell 2014). A notable exception is the translation initiation factor eIF4A and its orthologues that constitute the most minimal DEAD-box protein group containing just the two RecA domains. The tails that flank other DEAD-box proteins have diverse lengths and sequence composition but do not form additional structured domains. In some instances, these extensions were shown to directly regulate the ATPase activity of DEAD-box proteins. For example, Dbp5/DDX19 contains an N-terminal $\alpha$-helix that inhibits the basal ATPase activity by inserting itself between the two RecA domains and stabilizing an inactive conformation (Collins et al. 2009). This autoinhibition is relieved upon Gle1 and RNA binding (Montpetit et al. 2011; von Moeller et al. 2009).

A significant number of DEAD-box ATPases contain intrinsically disordered tails that have amino acid sequences with low complexity (low complexity regions, LCRs). These extensions are unusually rich in specific amino acids, such as glutamine, asparagine, glycine or 
serine, or contain blocks of cations like arginine and aromatic amino acids such as tyrosine that can promote cation-pi or pi-pi interactions (Borcherds et al. 2021; Martin and Holehouse 2020). Of the DEAD-box ATPases in budding yeast and humans, more than $1 / 3$ contain tails with LCRs, and 3 out the 5 DEAD-box proteins in E. coli share this feature as well (Hondele et al. 2019).

In the last few years, it became clear that such LCRs can engage in weak hetero- or homotypic protein-protein interactions and promote the formation of higher-order protein meshworks that above a certain concentration threshold undergo a process termed liquid-liquid phase separation (LLPS) (Choi et al. 2020; Hyman et al. 2014; Kato et al. 2012; Shin and Brangwynne 2017). LLPS is an equilibrium process in which biomolecules, like proteins or RNA, weakly associate with each other via multivalent interactions to form reversible condensates that manifest as liquid-like droplets within the surrounding solution. Following the law of mass action condensates begin to assemble when the concentration of one of their components reaches a saturation limit. The partitioning of insoluble components into droplets lowers the free energy of the system and can lead to their enrichment by several orders of magnitude over the surrounding environment. The process reaches equilibrium when there is sufficient accumulation within the condensate and the concentration of the soluble pool is again below the solubility threshold (Choi et al. 2020; Hyman et al. 2014; Shin and Brangwynne 2017).

Consistent with this concept, DEAD-box proteins that contain tails with LCRs, from, e.g., humans, C. elegans, yeast or E.coli, can undergo LLPS (Elbaum-Garfinkle et al. 2015; Hondele et al. 2019; Nott et al. 2015). The LLPS saturation limit of these DEAD-box proteins in vitro is usually in the low micromolar range roughly in line with their cellular concentrations (Hondele et al. 2019). Whereas the detailed biochemical conditions that trigger LLPS vary between different DEAD-box proteins, RNA and ATP often promote condensation, likely due to the fact that RNA provides a scaffold and increases the valency of the system. However, a critical element for the in vitro condensation behavior of DEAD-box proteins lies within the LCR tails since family members that lack LCRs have a dramatically reduced ability to undergo condensation and removal of LCRs inhibits LLPS (Hondele et al. 2019). Furthermore, for the yeast DEAD-box ATPase Dhh1/Ddx6, the N- and C-terminal extensions, which are asparagine and glutamine-rich, respectively, were shown to be necessary and sufficient for condensation in vitro (Hondele et al. 2019; Faltova et al. 2018).

\section{DEAD-box proteins are global regulators of RNA-containing membraneless organelles}

LLPS is not only an in vitro phenomenon. In the last few years it has become abundantly clear that LLPS plays important roles in the formation of membraneless organelles and in the organization of all cells, from bacteria to humans (Al-Husini et al. 2018; Hyman et al. 2014; Shin and Brangwynne 2017). Membraneless organelles and biomolecular condensates have been implicated in an evergrowing list of biological processes including, e.g., chromatin organisation and DNA segregation, as well as numerous RNA processing and gene expression events (Hooper and Hilliker 2013; Hyman et al. 2014; Shin and Brangwynne 2017; Yoshizawa et al. 2020). Such ubiquitous roles in both pro- and eukaryotic cells indicate that the process of LLPS may be evolutionary old, and that phaseseparated structures might have contributed to the compartmentalisation of biochemical reactions since early steps of life, potentially already in a primordial RNA world prior to the emergence of biological membranes (Hansma 2017; Hondele et al. 2020; Yoshizawa et al. 2020).

Many prominent cellular biomolecular condensates contain both proteins and RNAs. This includes the nucleolus, nuclear speckles, cytoplasmic processing bodies (PBs), germ granules or stress granules (SGs). In each of these membraneless organelles, DEAD-box ATPase are abundant components: numerous DEAD-box ATPases involved in rRNA processing can be detected in the nucleolus (Andersen et al. 2002), Sub2/UAP56 is prominently found in nuclear speckles (Kota et al. 2008), Vasa/ DDX4 enriches in germ granules (Hay et al. 1988), and Dhh1/DDX6 and Ded1/DDX3 are central components of PBs and SGs, respectively (reviewed in Hooper and Hilliker [2013]).

In budding yeast, PBs and SG only form during stress conditions when translation is attenuated. A first hint that DEAD-box ATPases might regulate the dynamics of these membraneless organelles came from experiments with ATP-hydrolysis deficient variants of Ded1 and Dhh1, which in unstressed cells induce either constitutive SGs or PBs (Carroll et al. 2011; Hilliker et al. 2011). Furthermore, tethering of Dhh1 to an mRNA is sufficient to target an mRNA to PBs and inhibiting Dhhl's ATPase activity, by mutations in either Dhh1 or its MIF4G-activator Not1, prevents PB turnover (Carroll et al. 2011; Mugler et al. 2016). This ATP-dependent condensation process can also be recapitulated in vitro, and 
Dhh1, in the presence of RNA and ATP, forms phase separated droplets that can be readily dissolved upon ATPase stimulation by Not1 (Mugler et al. 2016). Altogether, this shows that the ATPase cycle of Dhh1 is coupled to its cycling into and out of PBs and characterizes the ATPase activity of Dhh1 as a critical regulator of $\mathrm{PB}$ formation.

This paradigm can be extended to additional DEAD-box ATPases and membraneless organelles indicating that this protein family has a global role in the regulation of RNA-containing biomolecular condensates (Hondele et al. 2019). Thus, they can function as cellular 'condensases'. For example, inhibition of the ATPase activity of UAP56 enhances the size of nuclear speckles and causes the accumulation of mRNA in these nuclear condensates (Hondele et al. 2019). Furthermore, the ATPase activity of eIF4A works in combination with Ded1 to regulate the formation of SGs (Tauber et al. 2020), and DEAD-box proteins localize to ribonucleoprotein condensates in bacteria as well (Al-Husini et al. 2018; Hondele et al. 2019).

A simple model was proposed of how DEAD-box ATPases function as 'condensases' to regulate the dynamics of ribonucleoprotein condensates (Figure 2, [Hondele et al. 2019]). RNA and ATP binding to a DEAD-box ATPase promotes condensation, whereas ATP hydrolysis and RNA release suppresses LLPS and triggers the recycling of the enzyme. In the most minimal scenario, ATP-dependent RNA-binding to an LCR-containing DEAD-box protein is both necessary and sufficient to induce condensation by interactions that are mediated by the tails: scaffolding by the RNA or multimerization upon RNA binding increases the valency, lowering the saturation threshold of the DEAD-box ATPase, and phase separation ensues. Upon ATP hydrolysis, the affinity for RNA decreases again, which pushes the protein back into the soluble phase (Figure 2). Such a model is supported by in vitro phase separation experiments but also by the findings that, e.g., a tailless variant of Dhh1 suppresses PB formation whereas expression of an ATPase-deficient protein leads to constitutive PBs in budding yeast (Carroll et al. 2011; Hondele et al. 2019).

However, cellular biomolecular condensates are complex and contain, in addition to DEAD-box proteins, a multitude of factors that can promote LLPS in vitro and membraneless organelle formation in vivo (Shin and Brangwynne 2017; Yoshizawa et al. 2020). Furthermore, DEAD-box ATPases, such as Sub2/UAP56 and eIF4A, which lack LCR tails and do not have the ability to phase separate by themselves, also regulate membraneless organelles (Hondele et al. 2019; Tauber et al. 2020). Thus, DEAD-box ATPases can also act in trans on membraneless organelles. One possible mode of action includes a clamping mechanism, whereby the DEAD-box protein forms a stable, ATP-dependent complex with RNA providing a binding platform for additional factors that in turn trigger phase separation. Importantly, RNA-RNA interactions also play a critical role in the condensation of ribonucleoprotein complexes (A. Jain and Vale 2017; Khong et al. 2017; Langdon et al. 2018; Tauber et al. 2020; Van Treeck et al. 2018). Recently, an in vitro SG reconstitution assay was described, and in this system, SG assembly is nucleated by RNA multimerization as well (Begovich and Wilhelm 2020). It is therefore likely that the helicase activity of DEAD-box ATPases also negatively regulates condensation by unwinding stretches of doublestranded RNA on opposing RNA molecules. This could prevent RNA entanglement that might occur in granules at high RNA concentrations, and furthermore, will lead to a decrease in valency thereby enhancing the solubility of

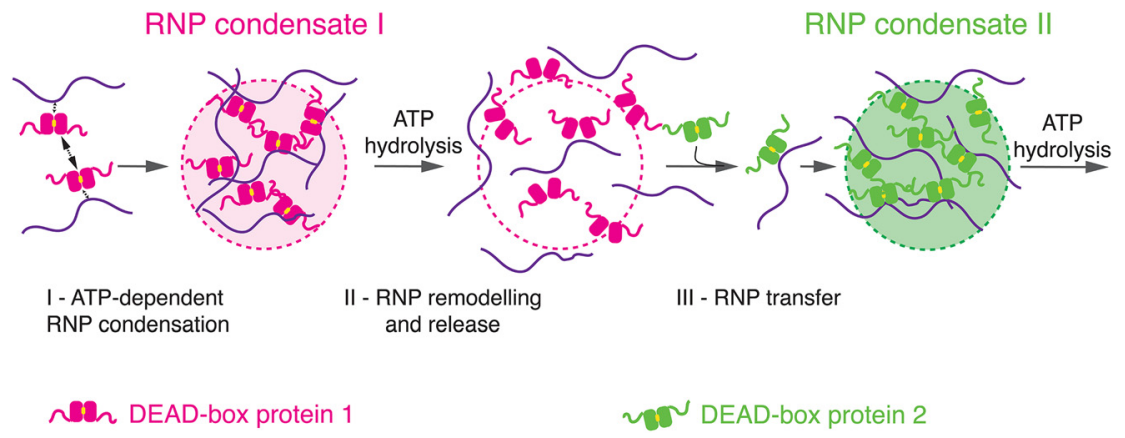

Figure 2: Model how DEAD-box ATPases function in RNA processing by regulating biomolecular condensation. ATP-dependent RNA binding of a DEAD-box protein leads to an increase in valency and the recruitment of cofactors triggering condensation of a ribonucleoprotein (RNP) complex (I). ATPase-driven remodeling of the RNP leads to compartment turnover and RNP release (II). The modified RNP interacts with the next DEAD-box protein and transfers to the next condensate (III). For details see text. Adapted from Hondele et al. (2019). 
ribonucleoprotein complexes (Begovich and Wilhelm 2020; Tauber et al. 2020).

It is important to point out that in addition to DEAD-box proteins, also other ATP consuming enzymes, play a critical role in the regulation of membraneless organelles. This includes kinases (e.g., Rai et al. [2018]; Ramachandran et al. [2011]; Shattuck et al. [2019]; and Wippich et al. [2013]) that can act as on/off switches in changing environments or during the cell cycle, or molecular chaperones (Cherkasov et al. 2013; S. Jain et al. 2016; Mateju et al. 2017) that use ATP to remodel protein assemblies.

\section{Functional role of DEAD-Box ATPases in membraneless organelles}

The structural arrangements that occur during the ATPase cycle of DEAD-box proteins were previously shown to mediate at least three biochemical activities. This includes a helicase activity to unwind RNA-RNA duplexes, a RNPase activity to remodel the protein composition of ribonucleoprotein complexes, and a clamping activity, in which DEAD-box proteins function as ATP-dependent scaffolds that recruit specific protein or RNA cofactors to RNAs (Jarmoskaite and Russell 2014). As discussed, a combination of all three of those functions is likely important to explain the role of DEAD-box ATPases in the regulation of biomolecular condensates. This could suggest a unifying view of the function of DEAD-box ATPases as cellular 'condensases' that control the assembly and turnover of ribonucleoprotein condensates. One central role of DEAD-box ATPases could be to define cellular reaction centres that locally concentrate both RNAs and interacting proteins in order to mediate distinct steps in the metabolism of RNA. Because of the evolutionary conservation of biomolecular condensation, such a 'condensase' function might even constitute the primordial role of DEAD-box proteins.

What could be the functional consequences of RNA recruitment to membraneless organelles? Biomolecular condensates are highly selective and can concentrate protein and RNA components by several orders of magnitude (Choi et al. 2020; Hyman et al. 2014; Shin and Brangwynne 2017). Hence, a DEAD-box ATPase-mediated targeting of an RNA to a condensate could keep this RNA away from soluble factors, to either hide or store it. This could make select groups of RNAs inaccessible for modifying enzymes or for the decay or translation machinery. Since cellular condensates were shown to be dynamic, an effective sequestration would however require low exchange rates and large partition coefficients for the affected RNAs. Conversely, very high local concentrations of decay or RNA modifying enzymes together with their RNA substrates in membraneless organelles could speed up reactions or lead to selective modification. For DEAD-box ATPases that could cause an increase in RNA unwinding or the remodeling of ribonucleoprotein complexes. Beyond concentration, the selective nature of LLPS could further aid with the remodeling activity of DEAD-box proteins, since targeting of an RNP to an organelle could enhance the removal of specific proteins if they are poorly miscible in the condensed phase (Hondele et al. 2020).

LLPS occurs when at least one of the components of a condensate crosses its solubility threshold, which occurs when the concentration of constituents rises, or more readily in a cellular context, when the strength or the valency of interactions increases. In cells, this can be accomplished by post-translational modifications as wells as by changes in crowding, fluidity, temperature or $\mathrm{pH}$ (Choi et al. 2020; Hondele et al. 2020; Hyman et al. 2014; Shin and Brangwynne 2017). The process continues until a new equilibrium is reached and the soluble concentration is again below the saturation limit (Choi et al. 2020; Hyman et al. 2014; Shin and Brangwynne 2017). Whereas components can passively exchange between condensates and the soluble phase, at thermodynamic equilibrium there is no directional net flux. However, living systems do not operate at equilibrium and the input of energy allows cells to keep biomolecular condensates 'alive', i.e., in an active, non-equilibrium state (Hondele et al. 2020; Hyman et al. 2014). This means that active condensates can generate biochemical work, and, e.g., enrich components against a concentration gradient, establish ribonucleoprotein states that are distinct inside or outside the organelle, generate molecular fluxes that can drive downstream reactions, or promote a directional translocation of RNP particles between compartments (Hondele et al. 2020).

The ATPase cycle of DEAD-box ATPases appears to provide critical energy input to keep RNA-containing membraneless organelles dynamic and away from equilibrium. How could this explain the functions of DEAD-box proteins in RNA metabolism? In an extreme model, one could envision that a dynamic network of DEAD-boxcontrolled membraneless compartments spatially and temporally controls cellular RNA processing reactions, akin to the membrane-enclosed secretory pathway, where proteins receive an ordered set of modifications when moving through the ER and distinct Golgi stacks (Figure 2). For example, mRNAs might travel through a series of condensates, from transcription and splicing to export 
through the nuclear pore, and ultimately to storage or decay. In each of these compartments, the mRNA and its associated protein coat will be modified by condensateenriched DEAD-box enzymes, RNA processing factors and RNA-binding proteins. The messenger ribonucleoprotein particle that leaves a compartment will be distinct from the one that entered, and such a DEAD-box ATPase-driven flux would control the fate of an mRNA throughout its life. Initial evidence in support of such a model comes from simple in vitro experiments but also from mutations in DEAD-box ATPases predicted to inhibit mRNA flux, which lead to a localized enrichment of mRNAs, e.g., at the site of transcription or in nuclear speckles (Hondele et al. 2019). However, it will now be critical to test this hypothesis thoroughly and examine whether DEAD-box ATPases function in a similar manner as 'condensases' also in other RNA maturation reactions and, for example, actively promote the flux of different rRNA processing intermediates through the different phases of the nucleolus to ensure the unidirectionality of this process.

\section{Outlook}

Membraneless organelles are widely used to organize the content of cells, yet the actual function of biomolecular condensation in many cellular processes remains rather poorly understood. However, we are gaining more and more insight into the pathways that promote and control condensate formation, which will provide us with better and more precise tools to influence the formation and dynamics of these cellular organization centres. Given their importance in many RNA processing reactions, the manipulation of DEAD-box ATPase proteins will continue to be valuable and will help us to unravel the function of cellular ribonucleoprotein condensates in RNA metabolism. In addition to addressing important cell biological problems, future research on DEAD-box ATPases may also further our understanding of how the misregulation of membraneless organelles can lead to pathological consequences since ribonucleoprotein granules have been implicated in the development of a large number of devastating aggregation diseases.

Acknowledgements: The author would like to thank all members of his laboratory for many fruitful discussions and enjoyable mini-meetings, and Maria Hondele for comments on the manuscript.
Author contributions: All the authors have accepted responsibility for the entire content of this submitted manuscript and approved submission.

Research funding: This work was supported by grants from the Swiss National Science Foundation (SNF 31003A_179275 and CRSII5_193740).

Conflict of interest statement: The authors declare no conflicts of interest regarding this article.

\section{References}

Al-Husini, N., Tomares, D.T., Bitar, O., Childers, W.S., and Schrader, J.M. (2018). $\alpha$-Proteobacterial RNA degradosomes assemble liquid-liquid phase-separated RNP bodies. Mol. Cell 71: 1027-1039.e14.

Alcazar-Roman, A.R., Tran, E.J., Guo, S., and Wente, S.R. (2006). Inositol hexakisphosphate and Gle1 activate the DEAD-box protein Dbp5 for nuclear mRNA export. Nat. Cell Biol. 8: 711-716.

Andersen, C.B.F., Ballut, L., Johansen, J.S., Chamieh, H., Nielsen, K.H., Oliveira, C.L.P., Pedersen, J.S., Séraphin, B., Le Hir, H., and Andersen, G.R. (2006). Structure of the exon junction core complex with a trapped DEAD-box ATPase bound to RNA. Science 313: 1968-1972.

Andersen, J.S., Lyon, C.E., Fox, A.H., Leung, A.K.L., Lam, Y.W., Steen, H., Mann, M., and Lamond, A.I. (2002). Directed proteomic analysis of the human nucleolus. Curr. Biol. 12: 1-11.

Barbosa, I., Haque, N., Fiorini, F., Barrandon, C., Tomasetto, C., Blanchette, M., and Le Hir, H. (2012). Human CWC22 escorts the helicase elF4AIII to spliceosomes and promotes exon junction complex assembly. Nat. Struct. Mol. Biol. 19: 983-990.

Begovich, K. and Wilhelm, J.E. (2020). An in vitro assembly system identifies roles for RNA nucleation and ATP in yeast stress granule formation. Mol. Cell 79: 991-1007.

Bono, F., Ebert, J., Lorentzen, E., and Conti, E. (2006). The crystal structure of the exon junction complex reveals how it maintains a stable grip on mRNA. Cell 126: 713-725.

Borcherds, W., Bremer, A., Borgia, M.B., and Mittag, T. (2021). How do intrinsically disordered protein regions encode a driving force for liquid-liquid phase separation? Curr. Opin. Struct. Biol. 67: 41-50.

Buchwald, G., Schüssler, S., Basquin, C., Le Hir, H., and Conti, E. (2013). Crystal structure of the human elF4AIII-CWC22 complex shows how a DEAD-box protein is inhibited by a MIF4G domain. Proc. Natl. Acad. Sci. U. S. A 110: E4611-E4618.

Cao, W., Coman, M.M., Ding, S., Henn, A., Middleton, E.R., Bradley, M.J., Rhoades, E., Hackney, D.D., Pyle, A.M., and De La Cruz, E.M. (2011). Mechanism of Mss116 ATPase reveals functional diversity of DEAD-box proteins. J. Mol. Biol. 409: 399-414.

Carroll, J.S., Munchel, S.E., and Weis, K. (2011). The DExD/H box ATPase Dhh1 functions in translational repression, mRNA decay, and processing body dynamics. J. Cell Biol. 194: 527-537.

Caruthers, J.M. and McKay, D.B. (2002). Helicase structure and mechanism. Curr. Opin. Struct. Biol. 12: 123-133. 
Chen, Y., Boland, A., Kuzuoğlu-Öztürk, D., Bawankar, P., Loh, B., Chang, C.-T., Weichenrieder, O., and Izaurralde, E. (2014). A DDX6-CNOT1 complex and W-binding pockets in CNOT9 reveal direct links between miRNA target recognition and silencing. Mol. Cell 54: 737-750.

Cherkasov, V., Hofmann, S., Druffel-Augustin, S., Mogk, A., Tyedmers, J., Stoecklin, G., and Bukau, B. (2013). Coordination of translational control and protein homeostasis during severe heat stress. Curr. Biol. 23: 2452-2462.

Choi, J.-M., Holehouse, A.S., and Pappu, R.V. (2020). Physical principles underlying the complex biology of intracellular phase transitions. Ann. Rev. Biophys. 49: 107-133.

Collins, R., Karlberg, T., Lehtiö, L., Schütz, P., van den Berg, S., Dahlgren, L.-G., Hammarström, M., Weigelt, J., and Schüler, H. (2009). The DEXD/H-box RNA helicase DDX19 is regulated by an $\alpha$-helical switch. J. Biol. Chem. 284: 10296-10300.

Elbaum-Garfinkle, S., Kim, Y., Szczepaniak, K., Chen, C.C.-H., Eckmann, C.R., Myong, S., and Brangwynne, C.P. (2015). The disordered $P$ granule protein LAF-1 drives phase separation into droplets with tunable viscosity and dynamics. Proc. Natl. Acad. Sci. U. S. A 112: 7189-7194.

Faltova, L., Küffner, A.M., Hondele, M., Weis, K., and Arosio, P. (2018). Multifunctional protein materials and microreactors using low complexity domains as molecular adhesives. ACS Nano 12: 9991-9999.

Folkmann, A.W., Noble, K.N., Cole, C.N., and Wente, S.R. (2011). Dbp5, Gle1-IP6 and Nup159. Nucleus 2: 540-548.

Hansma, H.G. (2017). Better than membranes at the origin of life? Life 7: 28.

Hay, B., Jan, L.Y., and Jan, Y.N. (1988). A protein component of Drosophila polar granules is encoded by vasa and has extensive sequence similarity to ATP-dependent helicases. Cell 55:577-587.

Henn, A., Cao, W., Hackney, D.D., and De La Cruz, E.M. (2008). The ATPase cycle mechanism of the DEAD-box rRNA helicase, DbpA. J. Mol. Biol. 377: 193-205.

Hilbert, M., Karow, A.R., and Klostermeier, D. (2009). The mechanism of ATP-dependent RNA unwinding by DEAD box proteins. Biol. Chem. 390: 1237-1250.

Hilbert, M., Kebbel, F., Gubaev, A., and Klostermeier, D. (2010). elF4G stimulates the activity of the DEAD box protein elF4A by a conformational guidance mechanism. Nucleic Acids Res. 39: 2260-2270.

Hilliker, A., Gao, Z., Jankowsky, E., and Parker, R. (2011). The DEAD-box protein Ded1 modulates translation by the formation and resolution of an elF4F-mRNA complex. Mol. Cell 43: 962-972.

Hondele, M., Heinrich, S., De Los Rios, P., and Weis, K. (2020). Membraneless organelles: phasing out of equilibrium. Emerg. Top. Life Sci. 4: 343-354.

Hondele, M., Sachdev, R., Heinrich, S., Wang, J., Vallotton, P., Fontoura, B.M.A., and Weis, K. (2019). DEAD-box ATPases are global regulators of phase-separated organelles. Nature 573: 144-148.

Hooper, C. and Hilliker, A. (2013). Packing them up and dusting them off: RNA helicases and mRNA storage. Biochim. Biophys. Acta Gene Regul. Mechan. 1829: 824-834.
Hyman, A.A., Weber, C.A., and Jülicher, F. (2014). Liquid-liquid phase separation in biology. Ann. Rev. Cell Dev. Biol. 30: 39-58.

Jain, A. and Vale, R.D. (2017). RNA phase transitions in repeat expansion disorders. Nature 546: 243-247.

Jain, S., Wheeler, J.R., Walters, R.W., Agrawal, A., Barsic, A., and Parker, R. (2016). ATPase-modulated stress granules contain a diverse proteome and substructure. Cell 164: 487-498.

Jarmoskaite, I. and Russell, R. (2014). RNA helicase proteins as chaperones and remodelers. Ann. Rev. 83: 697-725.

Kato, M., Han, T.W., Xie, S., Shi, K., Du, X., Wu, L.C., Mirzaei, H., Goldsmith, E.J., Longgood, J., Pei, J., et al. (2012). Cell-free formation of RNA granules: low complexity sequence domains form dynamic fibers within hydrogels. Cell 149: 753-767.

Khong, A., Matheny, T., Jain, S., Mitchell, S.F., Wheeler, J.R., and Parker, R. (2017). The stress granule transcriptome reveals principles of mRNA accumulation in stress granules. Mol. Cell 68: 808-820.

Kota, K.P., Wagner, S.R., Huerta, E., Underwood, J.M., and Nickerson, J.A. (2008). Binding of ATP to UAP56 is necessary for mRNA export. J. Cell Sci. 121: 1526-1537.

Langdon, E.M., Qiu, Y., Niaki, A.G., McLaughlin, G.A., Weidmann, C.A., Gerbich, T.M., Smith, J.A., Crutchley, J.M., Termini, C.M., Weeks, K.M., et al. (2018). mRNA structure determines specificity of a polyQ-driven phase separation. Science 360: 922-927.

Le Hir, H., Izaurralde, E., Maquat, L.E., and Moore, M.J. (2000). The spliceosome deposits multiple proteins 20-24 nucleotides upstream of mRNA exon-exon junctions. EMBO J. 19: 6860-6869.

Linder, P. and Jankowsky, E. (2011). From unwinding to clamping - the DEAD box RNA helicase family. Nat. Rev. Mol. Cell Biol. 12: 505-516.

Martin, E.W. and Holehouse, A.S. (2020). Intrinsically disordered protein regions and phase separation: sequence determinants of assembly or lack thereof. Emerg. Top. Life Sci. 18: 285-329.

Mateju, D., Franzmann, T.M., Patel, A., Kopach, A., Boczek, E.E., Maharana, S., Lee, H.O., Carra, S., Hyman, A.A., and Alberti, S. (2017). An aberrant phase transition of stress granules triggered by misfolded protein and prevented by chaperone function. EMBO J. 36: 1669-1687.

Mathys, H., Basquin, J., Ozgur, S., Czarnocki-Cieciura, M., Bonneau, F., Aartse, A., Dziembowski, A., Nowotny, M., Conti, E., and Filipowicz, W. (2014). Structural and biochemical insights to the role of the CCR4-NOT complex and DDX6 ATPase in MicroRNA repression. Mol. Cell 54: 751-765.

Montpetit, B., Thomsen, N.D., Helmke, K.J., Seeliger, M.A., Berger, J.M., and Weis, K. (2011). A conserved mechanism of DEAD-box ATPase activation by nucleoporins and InsP6 in mRNA export. Nature 472: 238-242.

Mugler, C.F., Hondele, M., Heinrich, S., Sachdev, R., Vallotton, P., Koek, A.Y., Chan, L.Y., and Weis, K. (2016). ATPase activity of the DEAD-box protein Dhh1 controls processing body formation. eLife 5: 4154.

Nielsen, K.H., Chamieh, H., Andersen, C.B.F., Fredslund, F., Hamborg, K., Le Hir, H., and Andersen, G.R. (2009). Mechanism of ATP turnover inhibition in the EJC. RNA 15: 67-75. 
Nott, T.J., Petsalaki, E., Farber, P., Jervis, D., Fussner, E., Plochowietz, A., Craggs, T.D., Bazett-Jones, D.P., Pawson, T., Forman-Kay, J.D., et al (2015). Phase transition of a disordered nuage protein generates environmentally responsive membraneless organelles. Mol. Cell 57: 936-947.

Oberer, M., Marintchev, A., and Wagner, G. (2005). Structural basis for the enhancement of elF4A helicase activity by elF4G. Genes Dev. 19: 2212-2223.

Ozgur, S., Buchwald, G., Falk, S., Chakrabarti, S., Prabu, J.R., and Conti, E. (2015). The conformational plasticity of eukaryotic RNA-dependent ATPases. FEBS J. 282: 850-863.

Pühringer, T., Hohmann, U., Fin, L., Pacheco-Fiallos, B., Schellhaas, U., Brennecke, J., and Plaschka, C. (2020). Structure of the human core transcription-export complex reveals a hub for multivalent interactions. eLife 9: e61503.

Rai, A.K., Chen, J.-X., Selbach, M., and Pelkmans, L. (2018). Kinasecontrolled phase transition of membraneless organelles in mitosis. Nature 559: 211-216.

Ramachandran, V., Shah, K.H., and Herman, P.K. (2011). The CAMP-dependent protein kinase signaling pathway is a key regulator of $P$ body foci formation. Mol. Cell 43: 973-981.

Russell, R., Jarmoskaite, I., and Lambowitz, A.M. (2014). Toward a molecular understanding of RNA remodeling by DEAD-box proteins. RNA Biol. 10: 44-55.

Schuller, S.K., Schuller, J.M., Prabu, J.R., Baumgärtner, M., Bonneau, F., Basquin, J., and Conti, E. (2020). Structural insights into the nucleic acid remodeling mechanisms of the yeast THO-Sub2 complex. eLife 9: 2620.

Schütz, P., Bumann, M., Oberholzer, A.E., Bieniossek, C., Trachsel, H., Altmann, M., and Baumann, U. (2008). Crystal structure of the yeast elF4A-elF4G complex: an RNA-helicase controlled by protein-protein interactions. Proc. Natl. Acad. Sci. U. S. A 105: 9564-9569.
Shattuck, J.E., Paul, K.R., Cascarina, S.M., and Ross, E.D. (2019). The prion-like protein kinase Sky1 is required for efficient stress granule disassembly. Nat. Commun. 10: 3614.

Shin, Y. and Brangwynne, C.P. (2017). Liquid phase condensation in cell physiology and disease. Science 357: eaaf4382.

Tauber, D., Tauber, G., Khong, A., Van Treeck, B., Pelletier, J., and Parker, R. (2020). Modulation of RNA condensation by the DEADbox protein elF4A. Cell 180: 411-426.

Van Treeck, B., Protter, D.S.W., Matheny, T., Khong, A., Link, C.D., and Parker, R. (2018). RNA self-assembly contributes to stress granule formation and defining the stress granule transcriptome. Proc. Natl. Acad. Sci. U. S. A 115: 2734-2739.

von Moeller, H., Basquin, C., and Conti, E. (2009). The mRNA export protein DBP5 binds RNA and the cytoplasmic nucleoporin NUP214 in a mutually exclusive manner. Nat. Struct. Mol. Biol. 16: 247-254.

Weirich, C.S., Erzberger, J.P., Flick, J.S., Berger, J.M., Thorner, J., and Weis, K. (2006). Activation of the DExD/H-box protein $\mathrm{Dbp} 5$ by the nuclear-pore protein Gle1 and its coactivator InsP6 is required for mRNA export. Nat. Cell Biol. 8: 668-676.

Wippich, F., Bodenmiller, B., Trajkovska, M.G., Wanka, S., Aebersold, R., and Pelkmans, L. (2013). Dual specificity kinase DYRK3 couples stress granule condensation/dissolution to mTORC1 signaling. Cell 152: 791-805.

Wong, E.V., Cao, W., Vörös, J., Merchant, M., Modis, Y., Hackney, D.D., Montpetit, B., and De La Cruz, E.M. (2016). P(I) release limits the intrinsic and RNA-stimulated ATPase cycles of DEAD-box protein 5 (Dbp5). J. Mol. Biol. 428: 492-508.

Yoshizawa, T., Nozawa, R.-S., Jia, T.Z., Saio, T., and Mori, E. (2020). Biological phase separation: cell biology meets biophysics. Biophys. Rev. 12: 519-539. 